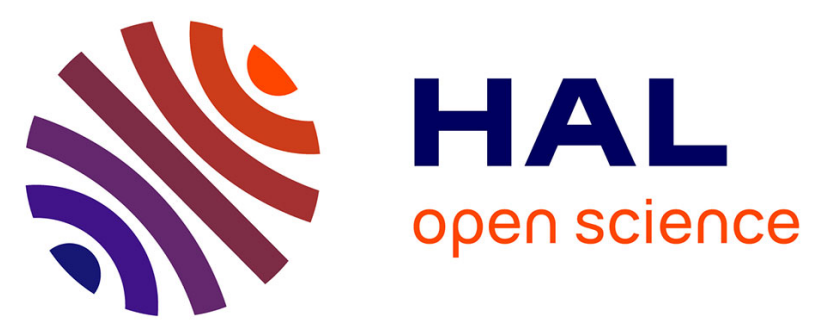

\title{
Investigation de divers procédés de texturation de céramiques supraconductrices à haute Tc
}

\author{
Pierre Régnier, Xavier Deschanels, Laurent Chaffron, Lélia Schmirgeld, Pol \\ Godelaine, Marcel Ausloos, Hyacinthe Vanderschueren, Robert Pollard, \\ Graham Mccartney, Clotilde Aguillon, et al.
}

\section{To cite this version:}

Pierre Régnier, Xavier Deschanels, Laurent Chaffron, Lélia Schmirgeld, Pol Godelaine, et al.. Investigation de divers procédés de texturation de céramiques supraconductrices à haute Tc. Journal de Physique III, 1992, 2 (2), pp.213-224. 10.1051/jp3:1992119 . jpa-00248736

\section{HAL Id: jpa-00248736 https://hal.science/jpa-00248736}

Submitted on 1 Jan 1992

HAL is a multi-disciplinary open access archive for the deposit and dissemination of scientific research documents, whether they are published or not. The documents may come from teaching and research institutions in France or abroad, or from public or private research centers.
L'archive ouverte pluridisciplinaire HAL, est destinée au dépôt et à la diffusion de documents scientifiques de niveau recherche, publiés ou non, émanant des établissements d'enseignement et de recherche français ou étrangers, des laboratoires publics ou privés. 


\title{
Investigation de divers procédés de texturation de céramiques supraconductrices à haute $\mathbb{T}_{\mathrm{c}}$
}

Pierre Régnier (1), Xavier Deschanels ( $\left.{ }^{1}\right)$, Laurent Chaffron ( $\left.{ }^{1}\right)$, Lélia Schmirgeld ( ${ }^{1}$ ), Pol Godelaine (2), Marcel Ausloos ( $\left.{ }^{2}\right)$, Hyacinthe Vanderschueren $\left({ }^{2}\right)$, Robert Pollard ( $\left.{ }^{3}\right)$, Graham McCartney $\left({ }^{3}\right)$, Clotilde Aguillon $\left({ }^{4}\right)$, Sadok Senoussi $\left({ }^{4}\right)$, Philippe Monod $\left({ }^{4}\right)$ et Nicole Bontemps $\left({ }^{4}\right)$

(1) DTA/CEREM/DTM/SRMP-CE Saclay, 91191 Gif-sur-Yvette Cedex, France

(2) Montefiore Univ. de Liège, Sart Tilman B-28, B 4000 Liège, Belgique

$\left({ }^{3}\right)$ M.S.E. The University of Liverpool, PO Box 147 Liverpool L 693 BX, G.B.

$\left({ }^{4}\right)$ Laboratoire de Physique des Solides, Université Paris-Sud, 91405 Orsay, France

(Reçu le 7 novembre 1990, accepté le 5 août 1991)

Résumé. - Nous avons étudié trois procédés de texturation des céramiques supraconductrices à haute température critique basés sur: l'alignement de grains paramagnétiques dans un champ magnétique intense, le frittage sous forte charge (frittage-fluage) et la solidification dirigée. Après avoir rappelé le principe et analysé les mécanismes contrôlant chacun de ces procédés, on présente les caractérisations microstructurales, magnétiques et électriques des meilleurs échantillons produits.

\begin{abstract}
We have investigated three processes of texturing high $T_{c}$ superconducting ceramics based on : alignment of paramagnetic grains in a high magnetic field, sintering under a heavy load (creep-sintering) and oriented solidification. After recalling the principle and analyzing the controling mechanisms of each process, microstructural, magnetic and electrical characterization of the best produced samples are presented.
\end{abstract}

\section{Introduction.}

Depuis plus d'un an, nous étudions en collaboration avec les Universités de Liège, de Liverpool et d'Orsay, les possibilités de texturer les céramiques supraconductrices à haute température critique par alignement magnétique, frittage-fluage et solidification dirigée.

\section{Alignement magnétique.}

On sait que placés dans un champ magnétique intense, des grains monocristallins de céramique supraconductrice à haute température critique toument sur eux-mêmes afin d'aligner leur direction de plus forte aimantation parallèlement au champ [1]. Vu que le tenseur de susceptibilité magnétique des céramiques de type $\mathrm{TRBa}_{2} \mathrm{Cu}_{3} \mathrm{O}_{7}$ est caractérisé par les deux valeurs principales $\chi_{\|_{c}}$ et $\chi_{\perp c}$, les grains, pourvu qu'ils soient libres, aligneront donc 
leur direction $c$ parallèlement au champ lorsque $\chi_{\|_{c}}>\chi_{\perp c}$, c'est le cas de TR $=$ Y, Ho et Dy à la température ambiante, et au contraire perpendiculairement au champ dans la situation opposée, c'est le cas de TR $=\mathrm{Er}, \mathrm{Eu}, \mathrm{Gd}$.

2.1 ANALYSE DU PHÉNOMĖNE. - Comme nous l'avons montré dans une analyse détaillée du phénomène [2], cette rotation des grains peut être prévue à partir de l'équation fondamentale de la dynamique. Le calcul montre que si au départ tous les grains sont orientés au hasard et que $\Delta \chi=\chi \|_{c}-\chi_{\perp c}$ est positif, alors environ $99,8 \%$ d'entre eux auront leur axe $c$ à moins de $1^{\circ}$ du champ appliqué, et que tous les autres grains seront quasiment orientés au hasard quand $t=10 \tau$. Pour fixer les idées, avec des grains monocristallins dispersés dans une résine époxy de viscosité 10 poises et placés à la température ambiante dans une induction de $1 \mathrm{~T}$, $\tau$ est d'environ $1 \mathrm{~s}$ pour 1'YBaCuO et $0,1 \mathrm{~s}$ pour le DyBaCuO.

2.2 Etude EXPÉRIMENTALE. - Pour étudier ce phénomène, nous avons dispersé $25 \%$ en poids de poudre de céramique à haute $T_{\mathrm{c}}$ dans $75 \%$ de résine d'enrobage et placé le tout dans un aimant permanent $(B=0,6 \mathrm{~T})$ ou dans une bobine supraconductrice $(B=5$ et $8 \mathrm{~T})$. Après 10 à $15 \mathrm{~min}$, la résine étant polymérisée, nous avons alors effectué des diagrammes de diffraction $X$ et caractérisé la désorientation des grains par le facteur de qualité

$$
Q=\frac{\text { Intensité de la raie }(110)}{\text { Intensité de la raie }(003)}
$$

$Q=0$ correspondant à une désorientation nulle de tous les grains et $Q=20$ à une désorientation quasiment aléatoire.

En passant de la poudre de DyBa $\mathrm{Cu}_{3} \mathrm{O}_{7}$ à travers une série de tamis aux mailles de plus en plus serrées, nous avons observé, toutes choses égales par ailleurs, que la désorientation des grains était d'autant plus faible que le tamis était plus fin (Fig. 1).

Comme d'après l'analyse précédente la rotation des grains est indépendante de leur taille, ce résultat laissait penser que la plupart des grains étaient multicristallins, hypothèse que nous avons vérifiée en observant la poudre en microscopie électronique à balayage. Afin de réduire la désorientation des grains, dans une seconde série d'expériences, nous avons compacté la poudre et refritté le matériau une centaine d'heures à $1000^{\circ} \mathrm{C}$, de manière à obtenir un bloc polycristallin, dont la taille moyenne des grains $(\sim 70 \mu \mathrm{m})$ soit supérieure à celle des mailles du tamis. Puis, nous avons rebroyé l'échantillon en poudre et tamisé cette dernière pour récolter les grains qui traversaient le tamis $20 \mu \mathrm{m}$ mais ne passaient pas à travers celui de $10 \mu \mathrm{m}$. En répétant l'expérience précédente avec cette poudre tamisée, nous avons abaissé le

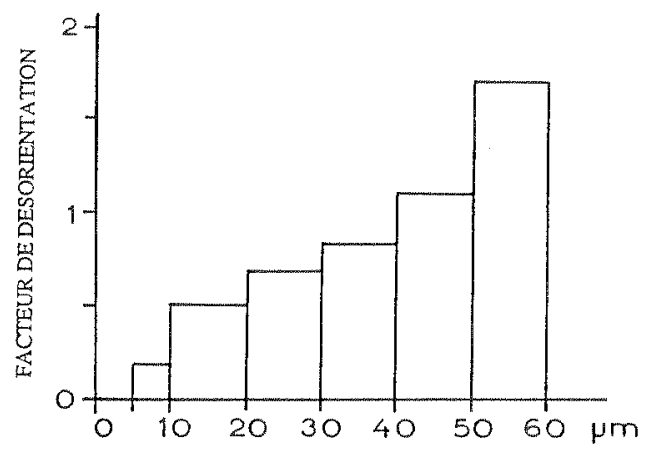

Fig. 1. - Variation du facteur de désorientation en fonction de la taille des mailles du tamis.

[Misorientation coefficient versus the sieve mesh.] 
facteur de désorientation jusqu'à $Q=0,0025$, toujours dans une induction de $0,6 \mathrm{~T}$; mais nous n'avons pu dépasser cette valeur même en utilisant des champs beaucoup plus forts (5 et 8 T) (Fig. 2). Ceci est vraisemblablement dû au fait que malgré le recuit de grossissement des cristallites, certains grains de poudre sont multicristallins. En effet, à haute température, les cristallites les plus grosses croissent au détriment des plus petites. De ce fait, il subsiste nécessairement des cristallites de très petite taille qui, lors du broyage, restent accolées à des fragments de grosses cristallites et constituent ainsi des grains multicristallins qui passent à travers les mailles des tamis.
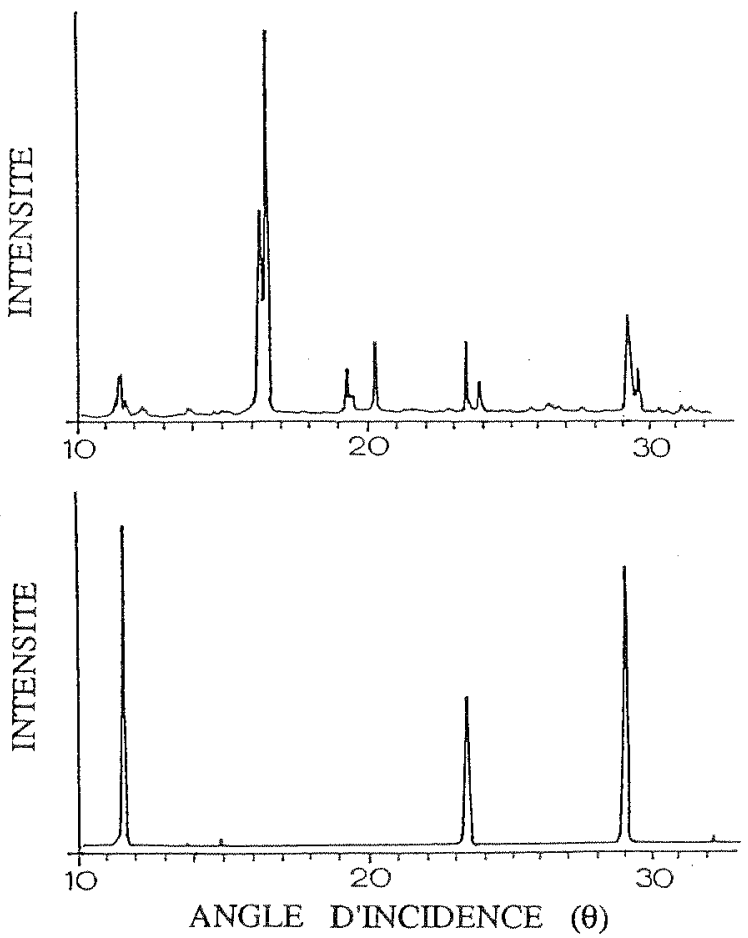

Fig. 2. - Diagrammes de diffraction $\mathrm{X}$ de céramique $\mathrm{DyBa}_{2} \mathrm{Cu}_{3} \mathrm{O}_{7}$. Haut : échantillon de référence non texturé ; Bas : échantillon le plus texturé obtenu par alignement des grains dans un champ de 0,6 T. $\left[\mathrm{X}\right.$ ray diffraction patterns of $\mathrm{DyBa}_{2} \mathrm{Cu}_{3} \mathrm{O}_{7-\delta}$ ceramics: Up: untextured sample. Down: the best textured sample obtained in a $0.6 \mathrm{~T}$ magnetic field.]

Par contre, comme on pouvait s'y attendre, avec de l' $\mathrm{YBa}_{2} \mathrm{Cu}_{3} \mathrm{O}_{7}$, nous avons trouvé que l'alignement des grains était nettement meilleur à $8 \mathrm{~T}$ qu'à $0,6 \mathrm{~T}(Q=0,04$ et 0,135 respectivement).

Le but de notre étude étant l'obtention de forts courants critiques par texturation du matériau, après avoir ainsi précisé les phénomènes et optimisé la qualité de la poudre, dans une seconde étape nous avons remplacé la résine isolante par différents liquides volatils: acétone, heptane, alcool... Après évaporation complète de ces derniers, nous avons recueilli une boue sèche que nous avons frittée $12 \mathrm{~h}$ à $920^{\circ} \mathrm{C}$ sous air, puis recuite $48 \mathrm{~h}$ à $400{ }^{\circ} \mathrm{C}$ sous oxygène. Ceci nous a conduit à des échantillons texturés $(0,1 \leqslant Q \leqslant 0,5$ avec $H=$ $0,6 \mathrm{~T})$ qui lévitaient à quelques millimètres au-dessus d'un aimant permanent refroidi à $77 \mathrm{~K}$, mais qui à cause de leur grande porosité étaient trop fragiles pour que l'on en relève les caractéristiques électriques. Nous en avons conclu qu'il était indispensable de compacter les 
grains avant frittage et avons conçu des dispositifs, en cours de réalisation, qui nous permettrons d'effectuer cette opération aussi bien sous champ que hors champ.

\section{Frittage-fluage.}

3.1 PRINCIPE DE LA MÉTHODE. - Nous appelons frittage-fluage l'opération qui consiste à fritter un échantillon sous une charge telle qu'il s'écrase abondamment en même temps qu'il se fritte. En l'absence de charge appliquée, on sait que la densification forte et rapide des céramiques à haute $T_{c}$, que l'on observe au-dessus de $900^{\circ} \mathrm{C}$, est due à la présence d'une phase liquide qui accélère les processus de frittage et qui confère aux grains leur forme de plaquettes parallèles au plan de base $(a, b)$ de la maille orthorhombique de leur structure cristalline. Par conséquent, si l'on écrase lentement les échantillons durant cette phase de frittage, il est clair que les grains, lubrifiés et entraînés par la phase liquide, rouleront et glisseront les uns sur les autres de manière à construire une structure ressemblant à un mur de briques, dans laquelle les plans conducteurs $a, b$ seront alignés. Mais il est aussi évident que la phase liquide accélérera la formation des ponts entre les grains qui s'opposeront à la rotation et au glissement de ces derniers. Par conséquent, la texturation des céramiques supraconductrices par frittage-fluage apparaît comme le résultat de deux tendances opposées et nécessite une optimisation pointue pour obtenir un haut niveau de texture.

3.2 OPTIMISATION DU PROCÉDÉ. - Afin d'obtenir les textures souhaitées, nous nous sommes efforcés d'optimiser le cycle de température et de charge représenté sur la figure 3 , en conservant constants les paramètres suivants: $T_{1}^{\circ}=600^{\circ} \mathrm{C} / \mathrm{h}, T_{1}=650{ }^{\circ} \mathrm{C}, \Delta t_{1}=0,5 \mathrm{~h}$, $\Delta t_{2}=1 \mathrm{~h}, \stackrel{\circ}{T}_{3}=60^{\circ} \mathrm{C} / \mathrm{h}, L_{1}=10 \mathrm{~kg}$ et sauf avis contraire, la vitesse d'écrasement $S$ durant la montée en charge. Tous les autres paramètres ont été variés systématiquement un à la fois.

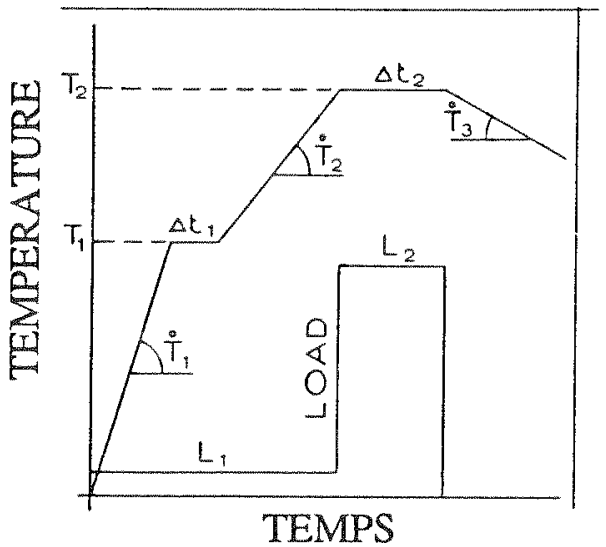

Fig. 3. - Cycle de température et de charge.

[Temperature and load schedule.]

Sur le plan pratique, nous avons découpé des tranches de $10 \mathrm{~mm}$ d'épaisseur dans des barreaux d'YBaCuO de $16 \mathrm{~mm}$ de diamètre obtenus par pressage à froid. Ces tranches prises en sandwich entre deux feuilles d'argent doublées de mica furent insérées dans les mâchoires d'une presse à chaud pour être frittées sous charge. Comme le montre la figure 4, pour une température de frittage donnée $\left(920^{\circ} \mathrm{C}\right)$, la désorientation des grains est d'autant plus faible que la charge appliquée est plus lourde. Mais, plus que la charge elle-même, ce qui importe 


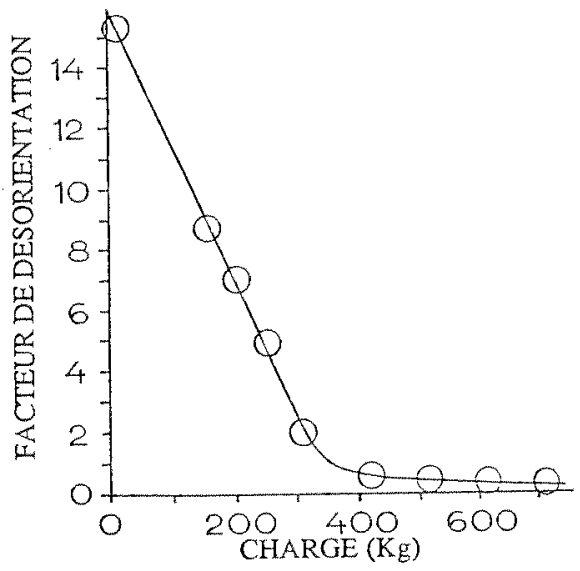

Fig. 4. - Influence de la charge sur la désorientation.

[Misorientation versus load.]

est le taux de déformation qu'elle impose à l'échantillon. En effet, même en faisant varier d'autres paramètres $\left(\stackrel{\circ}{T}_{2}, T_{2}, \Delta t_{2}, \ldots\right)$ nous avons constaté (cf. Fig. 5) que dans la représentation facteur de qualité - taux de déformation ( $\tau=$ épaisseur initiale/épaisseur finale), les points représentatifs de tous nos échantillons se situaient sur une même courbe et qu'il fallait réduire l'épaisseur des échantillons d'un facteur 10 environ pour obtenir une texture très prononcée à la fin du cycle schématisé sur la figure 1.

Les courbes de fluage que nous avons enregistrées montrent que la plus large part de la déformation s'effectue durant la courte période pendant laquelle on augmente la charge jusqu'à sa valeur nominale, mais que l'échantillon continue de se déformer à une vitesse de plus en plus lente tout au long du palier de frittage. Pour approfondir la compréhension des mécanismes de texturation, il importe donc de savoir quand se construit la texture : durant le court stade de déformation rapide ou au contraire au cours du long stade de fluage proprement dit. Afin d'éclairer ce point, nous avons effectué deux séries d'expériences. Dans la première, lors de la mise en charge, les échantillons furent écrasés à différentes vitesses

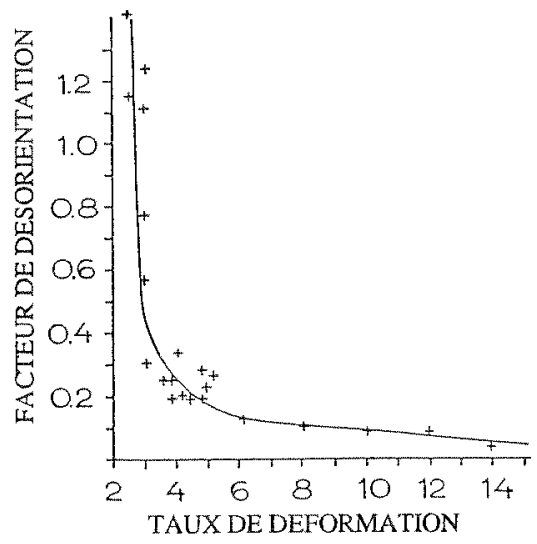

Fig. 5. - Influence du taux de déformation sur la désorientation des grains.

[Misorientation versus deformation rate.] 
jusqu'à ce que le taux de déformation atteigne 2,5, puis la charge fût enlevée immédiatement et les échantillons sortis du four aussi vite que possible. Les diagrammes de diffraction $X$ effectués sur ces échantilions montrent clairement qu'à la fin de ce stade de déformation rapide les échantillons sont déjà notablement texturés: $Q \sim 1$ si la vitesse de déformation est comprise entre 0,2 et $1 \mathrm{~mm} / \mathrm{min}$. Aux vitesses inférieures les ponts formés entre les grains durant la montée en température se consolident plus vite par frittage, qu'ils ne se déforment par fluage, ce qui freine la rotation des grains et réduit leur alignement. Aux vitesses supérieures, nous pensons que le transport de matière par la phase liquide n'est plus assez rapide pour donner aux grains leur forme de plaquette, ceux-ci se rangent donc de façon plus aléatoire et de ce fait, la texture est moins prononcée. Par ailleurs, nous avons remarqué que dans ce stade de déformation rapide la vitesse de déformation est proportionnelle à la contrainte appliquée à une puissance égale à 1 aux erreurs expérimentales près. Ceci indique que la vitesse n'est pas contrôlée par le glissement et la montée des dislocations car elle serait alors proportionnelle à la contrainte appliquée à une puissance comprise entre 3,5 et 7 , mais par un mécanisme d'écoulement visqueux.

Dans la seconde série d'expériences, nous avons conservé la vitesse d'écrasement constante $(5 \mathrm{~mm} / \mathrm{min})$ jusqu'à ce que la charge atteigne $300 \mathrm{~kg}$ et avons alors asservie la vitesse pour maintenir la charge constante. En utilisant un nouvel échantillon à chaque fois, nous avons observé la progression de la texture en fonction de la durée du frittage sous contrainte (Fig. 6). Cette courbe traduit assez bien le ralentissement rapide de la vitesse de déformation en fluage et corrobore la corrélation entre le facteur de désorientation et le taux de déformation (cf. Fig. 5).

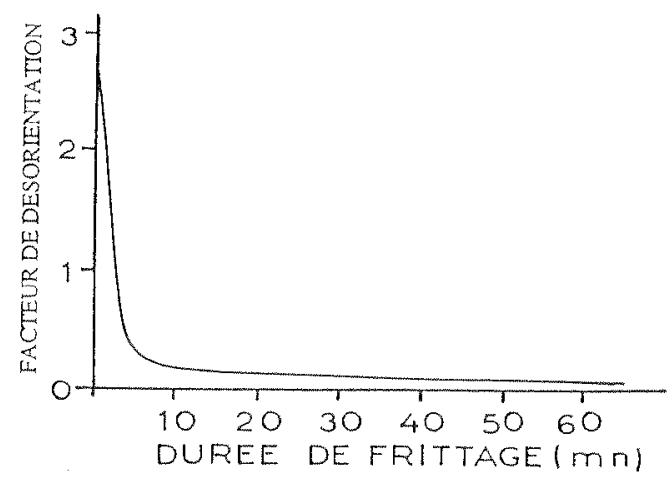

Fig. 6. - Evolution de la désorientation en fonction de la durée de frittage.

[Misorientation versus sintering time.]

L'effet de la température de frittage sur l'alignement des grains (Fig. 7) confirme pleinement l'idée d'un frittage en présence d'une phase liquide. En effet, pour une charge donnée $(310 \mathrm{~kg})$, nous avons observé qu'en dessous de $900^{\circ} \mathrm{C}$ l'échantillon ne se déformait quasiment pas et par suite ne se texturait pas $(Q>8)$, tandis que dès $910^{\circ} \mathrm{C}$ il commençait à se déformer et à se texturer $(Q \sim 3)$ et qu'à $920^{\circ} \mathrm{C}$ la déformation et la texture étaient très prononcées $(Q \leqslant 0,5)$. Il est clair qu'une dépendance aussi brutale de la vitesse de déformation avec la température est en parfait accord avec l'apparition d'une phase liquide vers $900^{\circ} \mathrm{C}$ et ne correspond à aucun mécanisme thermiquement activé connu. A plus haute température, la texture est moins bonne car la déformation est moindre vu que le pourcentage de phases secondaires augmente à partir de $920^{\circ} \mathrm{C}$, que l'on applique ou non une contrainte sur l'échantillon [3]. 


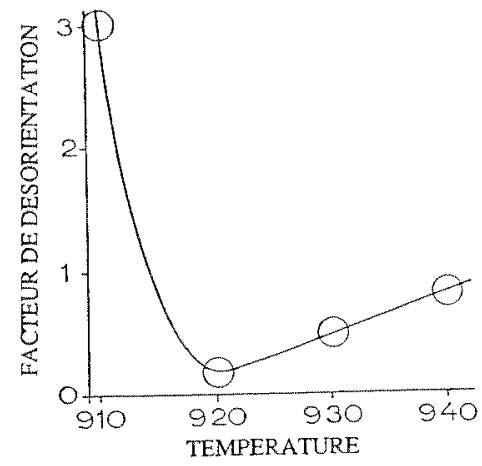

Fig. 7. - Influence de la température de frittage sur la désorientation des grains.

[Misorientation versus sintering temperature.]

D'une manière plus générale, comme nous l'avons montré dans des publications antérieures $[4,5]$, l'influence sur la qualité de la texture, de tous les autres paramètres : vitesse de montée en température; instant auquel on applique la charge; nature du matériau de contact... est en bon accord avec l'hypothèse d'un frittage en présence d'une phase liquide.

3.3 CARACTÉRISATION MICROSTRUCTURALE. - Nous avons systématiquement observé en microscopie optique des sections polies de nos échantillons, tant parallèles que perpendiculai-

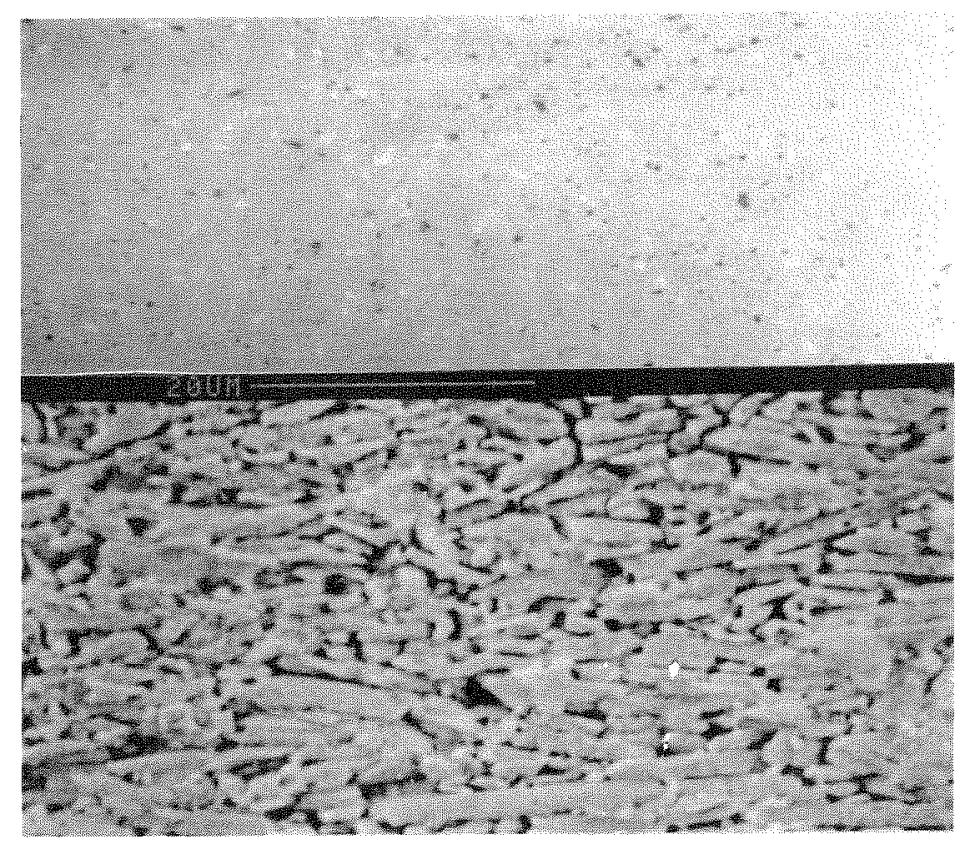

Fig. 8. - Aspect d'une coupe parallèle à la charge appliquée d'un échantillon texturé à $Q=0,05$ préparé avec de la poudre RP : a) microscopie optique lumière blanche, b) microscope à balayage après gravage des joints.

[Microstructure parallel to the load for a sample with $Q=0.05$. Rhone-Poulenc precursors. a) Optical microscope observation. b) SEM observation after etching.] 
res à la charge appliquée. En lumière ordinaire, sur tous les échantillons préparés à partir d'YBaCuO Rhône-Poulenc, on distingue seulement quelques grains brillants et d'autres plus petits et noirs que nous avons identifiés à la microsonde électronique comme étant respectivement du $\mathrm{CuO}$ et du $\mathrm{BaCuO}_{2}$. Ces échantillons semblent parfaitement compacts car aucun pore n'est visible à cette échelle (Fig. 8a). Au contraire avec la poudre P9, préparée au laboratoire, des pores sont visibles mais la porosité n'excède pas quelques \% (Fig. 8b). Ceci se comprend bien car la poudre RP est fine $(\sim 1 \mu \mathrm{m})$ incomplètement, réagie et carbonatée, tandis que la poudre P9 est grosse $(\sim 5 \mu \mathrm{m})$ et, nettement plus monophasée. Par suite, il y a plus de phase liquide avec la poudre RP qu'avec la poudre P9 et très logiquement la densification est plus forte avec RP qu'avec P9. En lumière polarisée les grains apparaissent sous forme de plaquettes, maclées ou non suivant le degré d'oxygénation, mais pour mieux révéler leur contour, nous avons gravé les joints de grains par attaque métallographique, ce qui permet en plus de les observer à fort grossissement au microscope à balayage. En optimisant les divers paramètres, avec la poudre RP nous avons atteint un facteur de qualité

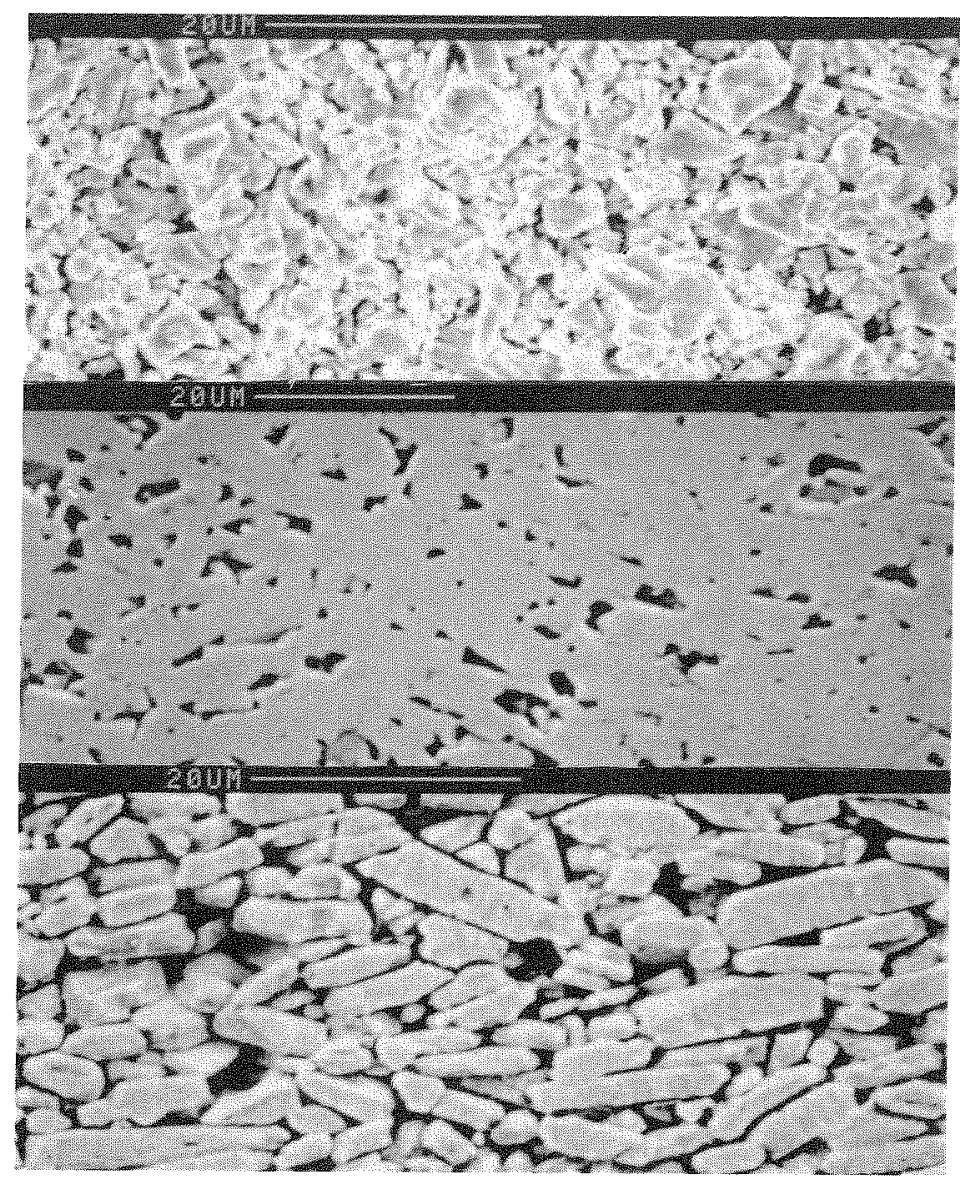

Fig. 9. - Observation en microscopie électronique à balayage d'une coupe parallèle à la charge appliquée d'un échantillon texturé à $Q=0,5$ préparé avec la poudre $\mathrm{P} 9$ : a) poudre compactée avant frittage, b) aspect après frittage sans gravage des joints, c) aspect après frittage et gravage des joints.

[SEM observations of microstructures parallel to the load for samples with $Q=0.5$. P9 powder. a) Green ceramic. b) After sintering, without etching. c) After sintering, with etching.] 
de 0,05 ce qui correspond à une désorientation de quelques degrés seulement entre les grains (cf. Fig. 8b).

Dans les mêmes conditions, avec la poudre P9, nous n'avons abaissé le facteur de désorientation que jusqu'à 0,5 . Comme le montrent les figures $9 \mathrm{a}, \mathrm{b}$ et c pour P9, mais cela reste vrai pour RP, le frittage-fluage s'accompagne d'une forte croissance des grains surtout de ceux qui sont les mieux orientés.

Au vu de ces observations, nous pensons que la texture se construit comme suit. Quand la phase liquide apparaît, elle mouille la surface des grains, au moins en partie, et s'accumule à leurs points de contact. Dès lors, les plus petits grains se dissolvent progressivement et la matière dissoute se redépose sur les plus gros. Ce phénomène, appelé coalescence, étant contrôlé par la diffusion dans une phase liquide, le flux de matière ainsi transportée est considérable et comme la vitesse de dépôt est très anisotrope, on conçoit que les grains acquièrent rapidement leur forme de plaquette parallèle aux plans $a, b$. A priori ces plaquettes sont rangées au hasard, mais en appliquant une contrainte uniaxiale, on les fait glisser et tourner sur elles-mêmes de manière à ce qu'elles forment une structure ressemblant à un mur de briques. Nous nous efforçons actuellement de modéliser ce phénomène très complexe.

3.4 CARACTÉRISATION ÉlECTRIQUE ET MAGNÉTIQUE. - En diffraction X, nous avons observé que bruts de frittage sous charge, tous les échantillons préparés avec la poudre RP ont une stœchiométrie en oxygène inférieure à 6,5 et de ce fait ne sont pas supraconducteurs, même à 4,2 K (d'après les mesures de susceptibilité alternative). Il est donc nécessaire de les réoxygéner. Mais probablement à cause de leur très forte densité cette opération est particulièrement difficile et nous n'y sommes parvenus qu'après un traitement de grossissement des grains qui a développé de la porosité secondaire. Ainsi traités, ces échantillons présentent une transition résistive très étroite à $92 \mathrm{~K}$ [6] et une anisotropie de susceptibilité magnétique comparable à celle observée sur les monocristaux (Fig. 10). Mais malgré la texture la densité critique de courant n'est que de $160 \mathrm{~A} / \mathrm{cm}^{2}$ à l'azote liquide car de nombreux joints sont soit fissurés soit bordés de phase amorphe provenant de la solidification de la phase liquide comme l'ont montré les observations en microscopie électronique en transmission.

Au contraire, bruts de frittage sous charge, les échantillons préparés avec la poudre P9 sont déjà légèrement supraconducteurs à $77 \mathrm{~K}$ et se réoxygènent raisonnablement bien. Les mesures de résistivité effectuées en courant continu sur des barreaux de $1,5 \times 1,5 \times$

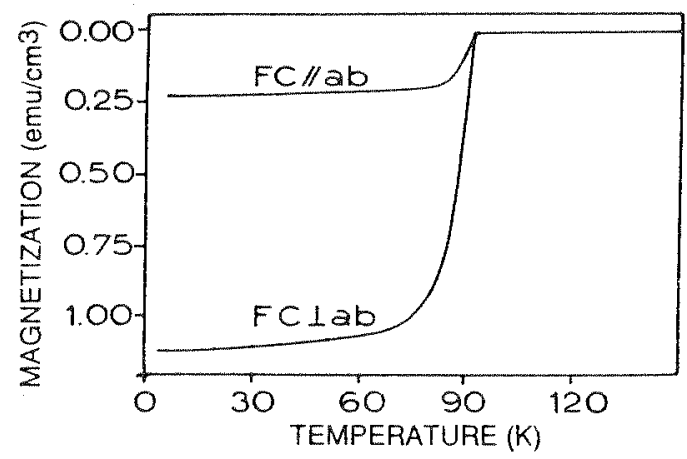

Fig. 10. - Variation de la magnétisation en fonction de la température mesurée en refroidissant les échantillons sous champ.

[Magnetization versus temperature during cooling in a magnetic field.] 


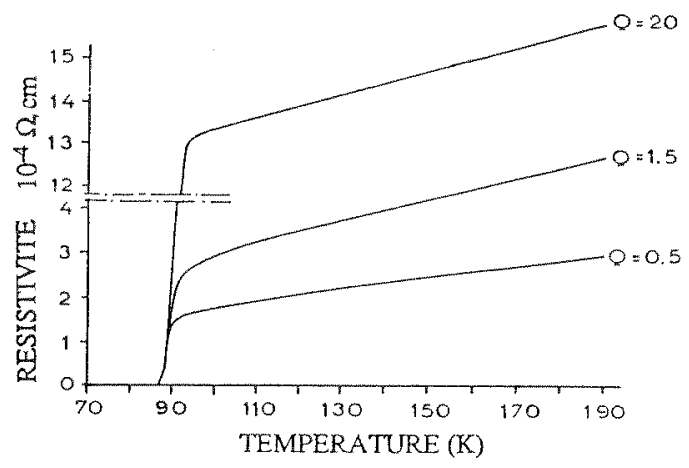

Fig. 11. - Variation de la résistivité en fonction de la température d'échantillons présentant des facteurs de désorientation différents.

[Resistivity versus temperature for samples with different $Q$ values.]

$20 \mathrm{~mm}^{3}$ par la méthode dite 4 points, montrent que dans le domaine normal la résistivité de nos échantillons est d'autant plus basse qu'ils sont plus texturés et que la transition résistive est d'abord très brutale vers $85 \mathrm{~K}$ puis beaucoup plus douce avant de s'annuler vers $80 \mathrm{~K}$ (Fig. 11). De ce fait le courant critique reste modeste : $J_{c}=600 \mathrm{~A} / \mathrm{cm}^{2}$ à $63 \mathrm{~K}$ mesuré sur l'échantillon présentant un facteur de désorientation de 1,5. En améliorant la texture, l'homogénéité du matériau et surtout la réoxygénation nous espérons largement dépasser ces valeurs.

Par ailleurs, nous avons obtenu des premiers résultats très encourageants avec les composés au bismuth qui ne nécessitent pas de réoxygénation.

\section{Solidification dirigée.}

Tous les supraconducteurs à haute $T_{\mathrm{c}}$ ont une fusion non congruante. Par conséquent, en portant ces matériaux au-dessus de leur point de fusion péritectique, on obtient une importante quantité de liquide en équilibre avec une quantité relativement faible de phase solide non supraconductrice (la phase verte 211 dans le cas des TRBaCuO). Si l'on refroidit très lentement le liquide dans des conditions très isothermes la vitesse de germination de la phase solide supraconductrice devient si lente que tout le liquide se solidifie sur les quelques germes formés et l'on obtient ainsi quelques gros cristaux. C'est la technique classique de préparation des petits monocristaux de supraconducteurs à haute $T_{\mathrm{c}}$ par la méthode dite du flux (on s'écarte volontairement de la composition de la phase supraconductrice pour conserver de la phase liquide en équilibre avec la phase solide). Au contraire, si on refroidit l'échantillon dans un faible gradient de température que l'on déplace lentement, on conserve une faible vitesse de germination et on oriente la croissance des grains dans le gradient de température. C'est la technique dite de melt texturing qui produit des échantillons fortement texturés présentant une densité critique de courant élevée [7]. Si le gradient est encore plus faible et qu'on le déplace encore plus lentement toute la phase liquide se solidifie sur le premier germe formé et on obtient une matrice monocristalline dans laquelle sont dispersés des grains de la phase non supraconductrice stable à haute température et le résidu de la phase liquide solidifiée. C'est la technique mise au point par nos partenaires de l'Université de Liverpool à partir des barreaux frittés d'YBaCuO que nous leur foumissons dans le cadre du contrat Brite/EURAM BE $0067 \mathrm{C}$ (EDB) dont nous sommes le leader. 
4.1 Caractérisation microstructurale. - Les barreaux qui ont 1,5 à $2 \mathrm{~mm}$ de diamètre sont monocristallins sur une longueur de $40 \mathrm{~mm}$. Comme l'orientation du premier germe est aléatoire les plans conducteurs $a, b$ sont généralement écartés de l'axe du barreau, typiquement 20 à $60^{\circ}$ dans les quelques échantillons préparés jusqu'à présent. Sur une coupe métallographique, on constate la même orientation sur toute la longueur de l'échantillon $(40 \mathrm{~mm})$. On observe de plus que les grains de phase verte 211 sont petits, très arrondis, qu'ils ne percolent pas et qu'ils n'occupent guère plus de 5 à $10 \%$ du volume. Quant au résidu de phase liquide qui s'est solidifié en dernier, il apparaît sous forme de strates discontinues distantes de 20 à $30 \mu \mathrm{m}$ et parallèles aux plans conducteurs.

4.2 CARACTÉRisation Électrique et MAGNÉtique. - En mesurant la chute de potentiel en fonction du courant continu traversant de façon permanente l'échantillon, jusqu'à près de $100 \mathrm{~A}$, nous avons déterminé que la densité critique de courant transporté correspondant à une chute de $1 \mu \mathrm{V} / \mathrm{cm}$ était de $7000 \mathrm{~A} / \mathrm{cm}^{2}$ à $77 \mathrm{~K}$ suivant l'axe du barreau. Par mesures magnétiques [8] rapportées à la longueur du barreau, nous avons confirmé cette valeur. De plus, en découpant des tranches d'échantillon parallèles aux plans conducteurs, nous avons déterminé le courant critique tant suivant l'axe $c$ que dans le plan $(a, b)$ par mesure magnétique en fonction de la température et du champ appliqué (Fig. 12).

En champ nul, la densité critique de courant dans le plan $(a, b)$ atteint $10^{5} \mathrm{~A} / \mathrm{cm}^{2}$ à $77 \mathrm{~K}$ et dépasse probablement $2 \times 10^{6} \mathrm{~A} / \mathrm{cm}^{2}$ à $4,2 \mathrm{~K}$, valeurs qui sont les meilleures rapportées actuellement sur des monocristaux massifs. Suivant l'axe $c$, ces valeurs tombent respectivement à $4 \times 10^{4}$ et $3 \times 10^{5} \mathrm{~A} / \mathrm{cm}^{2}$ ce qui reste très élevé.

Ce dernier faisceau de courbes montre la très bonne tenue au champ, au moins jusqu'à $3 \mathrm{~T}$, de la densité critique de courant de nos échantillons, aussi bien suivant l'axe $c$ que le long des plans $a, b$. On voit donc que ces échantillons de grande taille ont des caractéristiques égales et même supérieures à celles mesurées sur les meilleurs monocristaux de petite taille préparés jusqu'alors.

Finalement les mesures de susceptibilité alternative effectuées à l'Université de Liège montrent que le courant critique des échantillons de ce type n'est pas limité par des problèmes de jonction faible car les courbes donnant la partie réelle et la partie imaginaire de la susceptibilité alternative en fonction de la température ne se déplacent pas vers les hautes températures quand on réduit le champ alternatif utilisé pour les mesures.

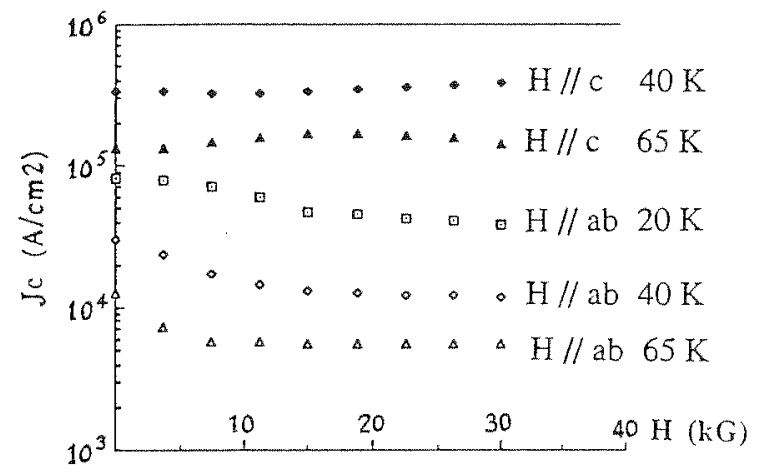

Fig. 12. - Variation de la densité critique de courant en fonction du champ appliqué parallèlement à $c$ ou parallèlement au plan $(a, b)$ à différentes températures.

$\left[J_{c}\right.$ versus magnetic field.] 


\section{Conclusion.}

Grâce à une bonne coopération entre physiciens du solide et scientifiques des matériaux, nous avons rapidement fait progressé notre étude sur la texturation des supraconducteurs à haute température critique. En poursuivant cet effort, nous espérons mieux comprendre tant les mécanismes qui contrôlent la formation des textures dans ces matériaux que les raisons physiques qui en limitent la densité critique de courant, ce qui devrait permettre d'optimiser ces matériaux pour les applications pratiques.

\section{Remerciements.}

Nous remercions la Communauté Economique Européenne pour le large soutien financier qu'elle nous accorde, l'Ecole des Mines d'Alès pour l'attribution d'une bourse ARMINE à Mr Deschanels, ancien élève de l'Ecole et le Comptoir Lyon Alemand Louyot pour le cofinancement de la bourse CIRCEA de Mr Chaffron. Nous remercions aussi Mrs Lesmond et Alloul de nous avoir prêté leurs bobines supraconductrices.

\section{Bibliographie}

[1] Farell D. E. and Chandrasekhar B. S., Phys. Rev. B 36 (1987) 4025.

[2] RéGnier P., Deschanels $X$. and FAisant J. P., à paraitre.

[3] Keating S. J., Wei I. and Chien T. Y., Ceramic Supraconductor II, Am. Ceram. Soc. (1988) 4350.

[4] Régnier P., Le Hazif R. and Chaffron L., Proceeding of the Int. Conf. on Modern Aspects of Superconductivity IITT International publication (Paris, 23-24 novembre 1989) pp. 133-138.

[5] Régnier P., Proceeding of the 7th CIMTEC World Ceramic Congress Satellite Conf. 4 HTC Superconductors (Trieste, 2-5 juillet 1990).

[6] Bonjour E. et al., Physica C 166 (1990) 451-455.

[7] Jin S., Sherwood R. C., Van Dover R. B., Tiefel T. H. and Johnson Jr D. W., Appl. Phys. Lett. 51 (1987) 203-204.

[8] Agullon C., MCCartney D. G., Régnier P. and Senoussi S., Submitted for publication to $J$. Appl. Phys. 